\title{
Tungsten Oxide Nanofibers for Electrochromic Device Application
}

\author{
${ }^{* 1}$ Cigdem Dulgerbaki, ${ }^{2}$ Ali Ihsan Komur, and ${ }^{2}$ Aysegul Uygun Oksuz \\ ${ }^{1}$ Alanya Alaaddin Keykubat University, Faculty of Engineering, Department of Engineering Fundamental \\ Sciences, 07450, Alanya, Antalya, Turkey \\ ${ }^{2}$ Suleyman Demirel University, Faculty of Arts and Science, Department of Chemistry, 32260 Isparta, Turkey
}

\begin{abstract}
The tungsten oxide $\left(\mathrm{WO}_{3}\right)$ nanofibers were grown directly onto an ITO-coated glass via an electrospinning method for electrochromic applications. The electrochromic properties of $\mathrm{WO}_{3}$ nanofibers were investigated in the presence of different electrolytes including a series of ionic liquids and classic $\mathrm{LiClO}_{4}-\mathrm{PC}$ system. A significant optical modulation of $20.82 \%$ at $760 \mathrm{~nm}$, reversible coloration with efficiency of $64.58 \mathrm{~cm}^{2} / \mathrm{C}$ and excellent cycling stability were achieved for the nanofiber electrochromic device (ECD) with ionic liquid based gel electrolyte.
\end{abstract}

Key words: Tungsten oxide, nanofiber, ionic liquid, electrochromic device

\section{Introduction}

Electrochromic materials can change their optical properties persistently and reversibly in the presence of a small electric difference [1]. It is widely accepted that the electrochromic phenomena of some inorganic materials is attributed to the injection/extraction of electrons and cations [2]. The kinetics and magnitude of ion insertion and the electrochromic reaction strongly depend on the diffusion length of ions and the available surface area, which is because the ratedetermining steps of ion intercalation and release are under diffusion control and are limited to a very thin surface layer of the host materials [3]. Therefore, it is important to design a material with unique architecture and proper crystal structure to obtain fast insertion kinetics and enhanced durability. [4]. The electrospinning process is becoming a powerful tool to obtain polymeric fibers with diameter in the nano-micron range. This electrostatic processing method uses a high-voltage electric field to form solid fibers from a polymeric fluid stream delivered through a millimeter-scale nozzle [5]. Recently, synthesis of one dimensional nanostructures; nanorods, nanowires or nanofibers have attracted much attention because these structures offer direct electrical pathways for electrons and can increase the electron transport rate [6]. In addition, they provide the vertically aligned nanostructure with a high surface area which allows the electrolyte to penetrate and shorten the proton diffusion paths within the bulk of material.

Tungsten trioxide $\left(\mathrm{WO}_{3}\right)$ has been identified as one of the most promising inorganic electrochromic materials [7]. $\mathrm{WO}_{3}$ electro-chromic systems including various morphologies such as thin films, nanosheets and nanorods have been fabricated through various methods including thermal evaporation, sol-gel, hydro-thermal route and layer-by-layer (LBL) technique. The tungsten trioxides thin films were deposited by thermal evaporation method onto indium tin oxides coated onto glass substrates. The coloration efficiency is found to be $31.25 \mathrm{~cm} 2 / \mathrm{C}$ for the as-deposited films and decreases to $18.3 \mathrm{~cm}^{2} / \mathrm{C}$ by annealing films at $723 \mathrm{~K}$ [8]. Electrochromic tungsten oxide $\left(\mathrm{WO}_{3}\right)$ thin films with nanometer-scale porosity have been synthesized via a solgel procedure making use of evaporation-induced self-assembly. Thus, only the $\mathrm{WO}_{3}$ films with a

*Corresponding author: Address: 'Alanya Alaaddin Keykubat University, Faculty of Engineering, Department of Engineering Fundamental Sciences, 07450, Alanya, Antalya, Turkey. E-mail address: cigdem.dulgerbaki@alanya.edu.tr, Phone: +902425106060 
highly crystalline framework exhibit almost unchanged electrochemical/electrochromic characteristics after prolonged potentiostatic cycling and exposure to elevated operating temperatures [9]. Nanobrick-like $\mathrm{WO}_{3}$ thin films have been synthesized via facile hydrothermal route. $\mathrm{WO}_{3}$ thin films were investigated to study optical attenuation and electrochromic properties. The transmittance variation of EC reaches about $28 \%$. The calculated CE value is $39.24 \mathrm{~cm}^{2} / \mathrm{C}$ [10]. Electrochromic films with a stacked structure of two-dimensional crystalline tungsten trioxide $\left(\mathrm{WO}_{3}\right)$ nanosheets were fabricated on fluorine-doped tin oxide (FTO) coated glass by a layer-by-layer (LBL) technique. Unique electrochromic property with a slow coloration process but a fast bleaching process was observed during the insertion/ extraction process of the protons into/from the stacked structure. The nanosheet film also exhibits a coloration efficiency of $32 \mathrm{~cm}^{2} / \mathrm{C}$ and an excellent long-term stability during the electrochromic process. The film exhibits an obvious transmittance difference in the visible light range with a maximum contrast of $48.5 \%$ at $800 \mathrm{~nm}$ [11].

Tungsten oxide films were deposited by a sol-gel method onto flexible substrates. Specific coatings were formed on PET:ITO substrates by using chloroalkoxides precursors. Optical contrast of the films was $20 \%$ at $630 \mathrm{~nm}$ between the bleached state at $+0.3 \mathrm{~V}$ and the colored state at $-1.2 \mathrm{~V}$, the coloration efficiency was found to be $36 \mathrm{~cm}^{2} / \mathrm{C}$ [12]. Uniform crystalline WO3 nanorods were synthesized by a facile hydrothermal process with $\mathrm{Na}_{2} \mathrm{WO}_{4} 2_{2} \mathrm{H}_{2} \mathrm{O}$ as the tungsten source and $\mathrm{NaCl}$ as the capping agent. The nanorods showed fast electrochromic switching response, comparable contrast and coloration efficiency, and much higher charge density (114.5 $\mathrm{mC} \mathrm{cm} \mathrm{mg}^{-1}$ ) than conventional amorphous $\mathrm{WO}_{3}$ films. It can be calculated that the maximum contrast of 54.9\% occurs at the wavelength of $800 \mathrm{~nm}$ [13]. However, no one has yet prepared tungsten oxide nanofibers as an electrochromic layer on a transparent conducting substrate for application in electrochromic devices (ECDs). In the present work, we described a simple method to prepare isolated tungsten oxide nanofibers directly grown on an ITO-coated glass. The nanofibers based electrochromic device was fabricated to demonstrate the potential in the electrochromic application with an ionic liquid based gel electrolyte and to compare with classical ACN:PC:PMMA:LiClO 4 electrolyte system. The optical and electrochromic performance, such as the contrast, stability etc. of the tungsten oxide nanofibers were extensively studied. The novelty of the present study is to assemble an ECD with $\mathrm{WO}_{3}$ nanofiber which does not exist in literature as far as our the best knowledge, thereby enhancing (tuning) $\mathrm{WO}_{3}$ 's electrochromic properties.

\section{Materials and Method}

\subsection{Chemicals and Materials}

Tungsten nanoparticles (W, 99.9\%, 40-60 nm), was supplied by SkySpring Nanomaterials. Hydrogen peroxide solution $\left(\mathrm{H}_{2} \mathrm{O}_{2}, 30 \%\right)$, ethanol $\left(\mathrm{C}_{2} \mathrm{H}_{5} \mathrm{OH}, 99.8 \%\right)$, Poly(ethylene oxide) (PEO, Mw 600,000) were obtained from Sigma-Aldrich and used as received.

\subsection{Preparation of tungsten oxide nanofibers}

A primary solution was prepared by dissolving $0.1 \mathrm{~g}$ of tungsten nanopowder in $1 \mathrm{ml}$ of $\mathrm{H}_{2} \mathrm{O}_{2}$ (30\%), $3 \mathrm{ml}$ ethanol (99.8\%), and $7 \mathrm{ml}$ deionized water $\left(\mathrm{H}_{2} \mathrm{O}\right)$. After long time stirring $(6 \mathrm{~h})$, the powder dissolved completely and a faint greenish-yellow solution was obtained. Poly(ethylene oxide) (PEO) was used as supporting polymer. Then $4 \mathrm{~g}$ deionized water and $0.2 \mathrm{~g}$ poly(ethylene 
oxide) were stirred for 2 days. The $\mathrm{WO}_{3}$ solution was then, mixed with the PEO solution to yield the optimized volume ratio of 3:1 $\left(\mathrm{WO}_{3} / \mathrm{PEO}\right)$. The resulting mixture was stirred for about 2 days at room temperature to produce a homogenous solution for electro- spinning. Fig. 1 shows a schematic diagram of the electrospinning setup used to produce nanofibers. In the electrospinning set up, about $2 \mathrm{ml}$ of polymer solutions was loaded into a $2 \mathrm{ml}$ syringe, equipped with a 21 gauge, stainless steel, a flat tip needle connected to a high-voltage supply (EMCO 4300). During the process, the solution was fed at a constant rate of $15 \mathrm{ml} / \mathrm{h}$ by a syringe pump (New Era Pump System Inc., USA). An electrical potential of $15 \mathrm{kV}$ was then applied between the tip of the needle with indium tin oxide glass target on the grounded aluminum foil which were $15 \mathrm{~cm}$ apart. Annealing of the obtained electrospinning fibers film onto indium tin oxide glass in the air at 500 _ $\mathrm{C}$ for $1 \mathrm{~h}$ was used to prepare $\mathrm{WO}_{3}$ nanofibers. Calcination process was responsible for the removal of $\mathrm{PEO}$ component and the growth of $\mathrm{WO}_{3}$ nanofiber.

\subsection{Measurement and characterization}

Surface morphologies of the $\mathrm{WO}_{3}$ fibers were observed by scanning electron microscopy (SEMEVO LS10 ZEISS). The chemical compositions were evaluated using energy dispersive X-ray (EDX). In order to examine the electrochromic behavior of $\mathrm{WO}_{3}$ nano-fibers, electrochemical measurements were performed with a three electrode measurement system into $1 \mathrm{M} \mathrm{LiClO}_{4}-\mathrm{PC}$ and four different ionic liquids 1-butyl-3-methylimidazoliumtetrafluoroborate (BMIMBF 4$), 1$ butyl-3-methylimidazolium hexafluorophosphate $\left(\mathrm{BMIMPF}_{6}\right)$, 1-butyl-3-methylimidazolium bis (trifluoromethylsulfonyl) imide (BMIMTFSI), and 1-butyl-1-meth- ylpyrrolidinium bis(trifluoromethylsulfonyl) imide (BMPTFSI). For cyclic voltammetry (CV) measurements, ITO coated $\mathrm{WO}_{3}$ fibers were used as working electrode, Pt wire was used as the counter electrode, and a $\mathrm{Ag} / \mathrm{AgCl}$ as the reference electrode. The redox behaviors of the $\mathrm{WO}_{3}$ fibers between +2.0 and $2.0 \mathrm{~V}$ were determined by using a Gamry $300 \mathrm{Model}$ potentiostat at a scan rate of $100 \mathrm{mV} \mathrm{s}^{-1}$.

\subsection{Gel electrolyte preparation and device fabrication}

For preparing the transparent gel polymeric electrolyte layer, polymethyl methacrylate (PMMA) was dissolved in propylene carbonate (PC) at room temperature, BMIMTFSI was added and the solution was magnetically stirred for $3 \mathrm{~h}$ at $70{ }^{0} \mathrm{C}$. The gel electrolyte consisted of BMIMTFSI/PMMA/PC in a weight ratio of 10:23:67 [14]. The resulting hot, homogeneous, transparent, viscous solution was immediately transferred into a clean glass petridish and it was left undisturbed for $24 \mathrm{~h}$. For comparison, second gel electrolyte based on ACN:PC:PMMA:LiClO 4 in weight ratio 70:20:7:3 was prepared. In order to carry out optical and electrochromic measurements, ECDs were fabricated. The ECDs fabricated is a sandwich structure, which contains two electrodes with electrolyte between them. $\mathrm{A} \mathrm{WO}_{3}$ nanofiber based electrode was employed as electrochromic electrode, and another piece of ITO glass sheet with the same size was employed as counter- electrode. Gel electrolyte (BMIMTFSI/PMMA/PC) or (ACN:PC:PMMA:LiClO 4 ) was infused between the two electrodes. Optical transmittance spectra were measured using a spectrophotometer (Ocean optics HR 4000, Mikropack Halogen Light Source HL-2000- FHSA in the 200-1000 nm wavelength range). 


\section{Results}

A surface SEM image of as-electrospun PEO/tungsten oxide fibers is shown in Fig 1, demonstrating randomly oriented nanofibers. The nanofibers were readily formed from the mixture solution of tungsten powder, hydrogen peroxide and PEO via electrospinning. The diameters of the composite nanofibers were distributed in the range of 30-100 $\mathrm{nm}$. A comparable surface SEM image was obtained for the sample thermally-treated at $500{ }^{0} \mathrm{C}$ in air (see Fig. $2 \mathrm{~b}$ ). There were distinct differences in surface morphology as the annealed nanofibers had rough surfaces. These differences may be attributed to the calcination of organic PEO from the as prepared composite nanofibers. In addition, amorphous tungsten oxide materials might aggregate to produce crystalline nanoparticles located along the 1-D direction to form a nanofiber with a rough surface during the calcination process [15].

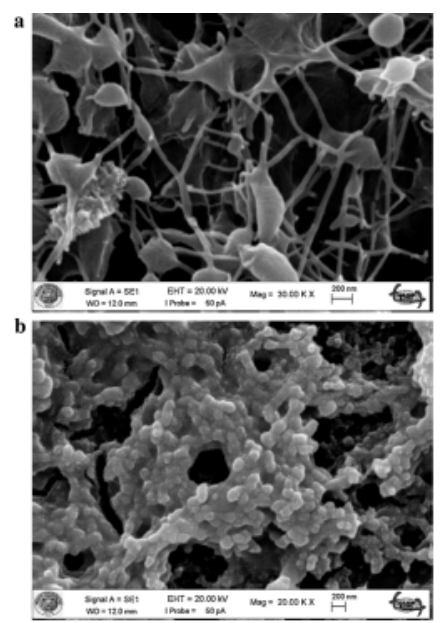

Figure 1. (a) SEM images of as-electrospun $\mathrm{WO}_{3} / \mathrm{PEO}$ composite nanofibers. (b) $\mathrm{WO}_{3}$ nanofibers calcined at $500{ }^{\circ} \mathrm{C}$ for $1 \mathrm{~h}$.

Electrochemical studies were performed in order to understand the electrochromic properties of nanostructured $\mathrm{WO}_{3}$ fibers. The cyclic voltammograms $(\mathrm{CV})$ were recorded for $\mathrm{ITO} / \mathrm{WO}_{3}$ fibers(working electrode) using three electrode cell configuration with platinum wire as counter, $\mathrm{Ag} / \mathrm{AgCl}$ as reference electrodes. Different ionic liquids; $\mathrm{BMIMBF}_{4}, \mathrm{BMIMPF}_{6}, \mathrm{BMIMTFSI}$ BMPTFSI and $1 \mathrm{M} \mathrm{LiClO}_{4}-\mathrm{PC}$ were used as electrolytes. The ionic liquids have been put in a wide range of synthetic applications as green solvents during the past decade for their good chemical and physical properties such as high ionic conductivity, fast ion mobility during redox events, low volatility, non-flammability and possess excellent thermal and chemical stability [16]. Cyclic voltammograms are shown in Fig. 2. When the potential was stepped from +2 to _2 $\mathrm{V}$, simultaneous intercalation of positive ions and electrons into $\mathrm{WO}_{3}$ films causes reduction of $\mathrm{W}^{6+}$ ions to lower valance $\mathrm{W}^{5+}$ ions and hence the film gets colored [17]. In ionic liquids, color changes are mainly attributed to the migration of counter ions from the electrolyte to the electrode surface [18]. The electrochromic color change of $\mathrm{WO}_{3}$ nanofiber is shown for +2 and $2 \mathrm{~V}$ in Fig. 3. During anodic potential scan, deintercalation of counter ions and electrons causes the bleaching of films and nanofiber appears transparent. The colored electrochromic fiber layer 
can retain its colors for several days after the applied voltages were removed. It is promising that significant energy-saving can be realized using this kind of electrochromic fiber [13].

The cathodic current, starting at approximately $+0.1 \mathrm{~V}$, is associated with the coloring process of $\mathrm{WO}_{3}$ nanofiber for all electrolytes (Fig. 2a-e). It is interesting to note that in BMIMTFSI and LiPC electrolytes shapes of cyclic voltammograms are similar and sharp and intense peaks were obtained. In other ionic liquids; $\mathrm{BMIMBF}_{4}, \mathrm{BMIMPF}_{6}$ and BMPTFSI, cyclic voltammograms are broader shaped. This may suggest that BMIMTFSI and Li-PC media offer an easy way to diffusion and charge transfer process of ions in $\mathrm{WO}_{3}$ fiber. [19]. This is primarily attributed to the lower viscosity of BMIMTFSI (54 cP) compared to BMIMBF4 (219 cP), BMIMPF6 (450 cP) or BMPTFSI $(85 \mathrm{cP})$ respectively, at $25{ }^{0} \mathrm{C}$ which is likely to relate to faster ion/molecule transport kinetics. Ionic liquids have high viscosities than Li-PC medium. Less viscous electrolyte allows faster diffusion of ions through the solution to the electrode surface leading to increase in current values [20].

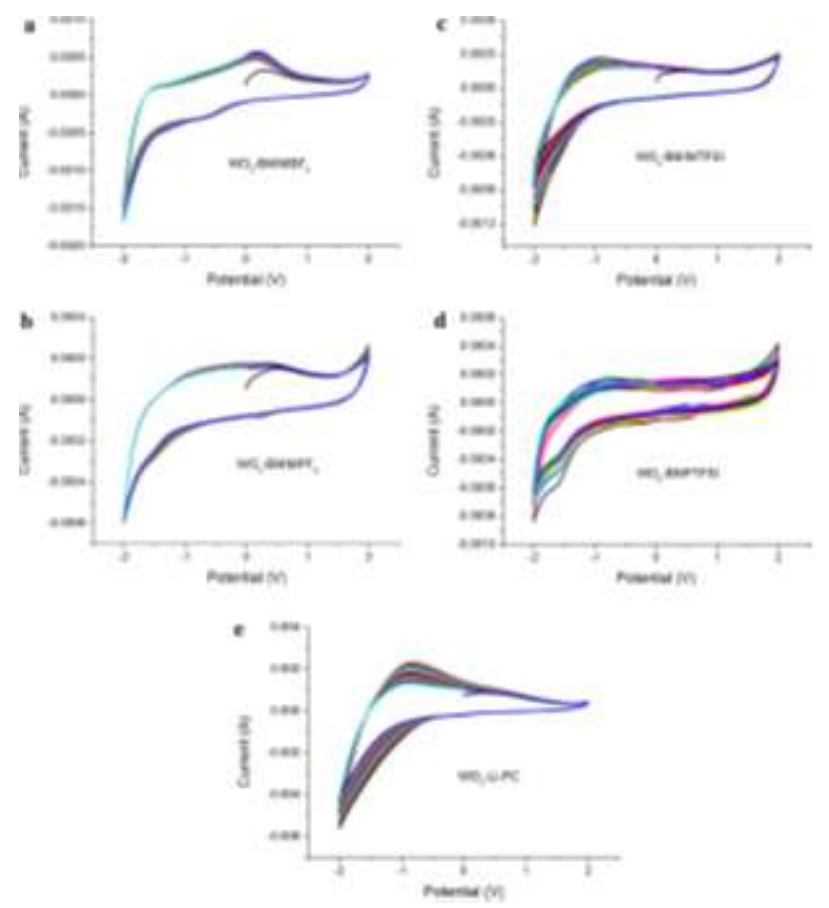

Figure 2. (a) Cyclic voltammograms of $\mathrm{WO}_{3}$ nanofibers in $\mathrm{BMIMBF}_{4}$ during 20 cycles at a scan rate of $100 \mathrm{mV} / \mathrm{s}$. (b) Cyclic voltammograms of $\mathrm{WO}_{3}$ nanofibers in $\mathrm{BMIMPF}_{6}$ during 20 cycles at a scan rate of $100 \mathrm{mV} / \mathrm{s}$. (c) Cyclic voltammograms of $\mathrm{WO}_{3}$ nanofibers in BMIMTFSI during 20 cycles at a scan rate of $100 \mathrm{mV} / \mathrm{s}$. (d) Cyclic voltammograms of $\mathrm{WO}_{3}$ nanofibers in BMPTFSI during 20 cycles at a scan rate of $100 \mathrm{mV} / \mathrm{s}$. (e) Cyclic voltammograms of $\mathrm{WO}_{3}$ nanofibers in $1 \mathrm{M} \mathrm{Li}-\mathrm{PC}$ during 20 cycles at a scan rate of $100 \mathrm{mV} / \mathrm{s}$.

An ECD was assembled employing $\mathrm{WO}_{3}$ nanofiber based electrode as electrochromic electrode as mentioned above. The color change of the ECD was evaluated using spectroelectrochemistry. Fig. 4a shows the optical transmittance spectra of the ECD with BMIMTFSI/PMMA/PC gel electrolyte in the UV-visible range under different driving voltages $(+3.0$ and $3.0 \mathrm{~V}$ vs. counter- electrode). It obtained its bleached state (transparent) at $+3.0 \mathrm{~V}$ and colored state (light blue) at $-3.0 \mathrm{~V}$. As observed in the same graph, the maximum gap of transmittance between two 
states is $20.82 \%$ and occurred at $760 \mathrm{~nm}$. Optical transmittance spectra of the ECD with ACN:PC:PMMA:LiClO 4 gel electrolyte is shown in Fig 4b. Transmittance difference was found as $4.25 \%$.
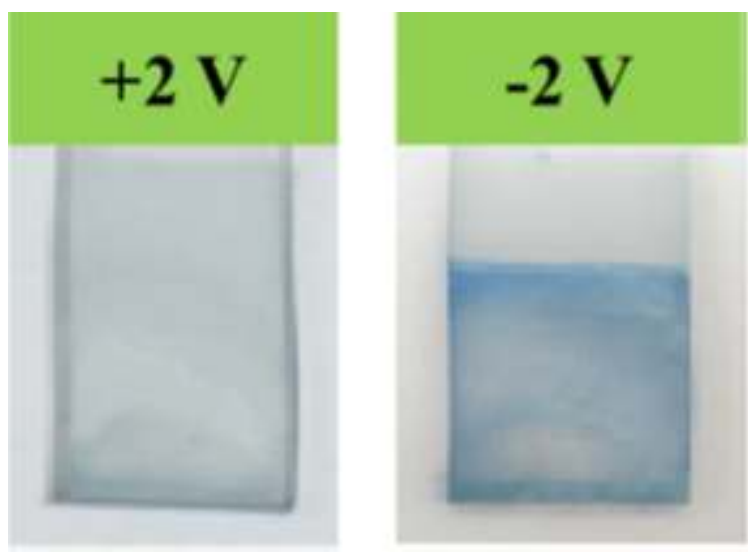

Figure 3. Photographs of $\mathrm{WO}_{3}$ nanofiber under $+2 \mathrm{~V}$ and $-2 \mathrm{~V}$ applied potentials.
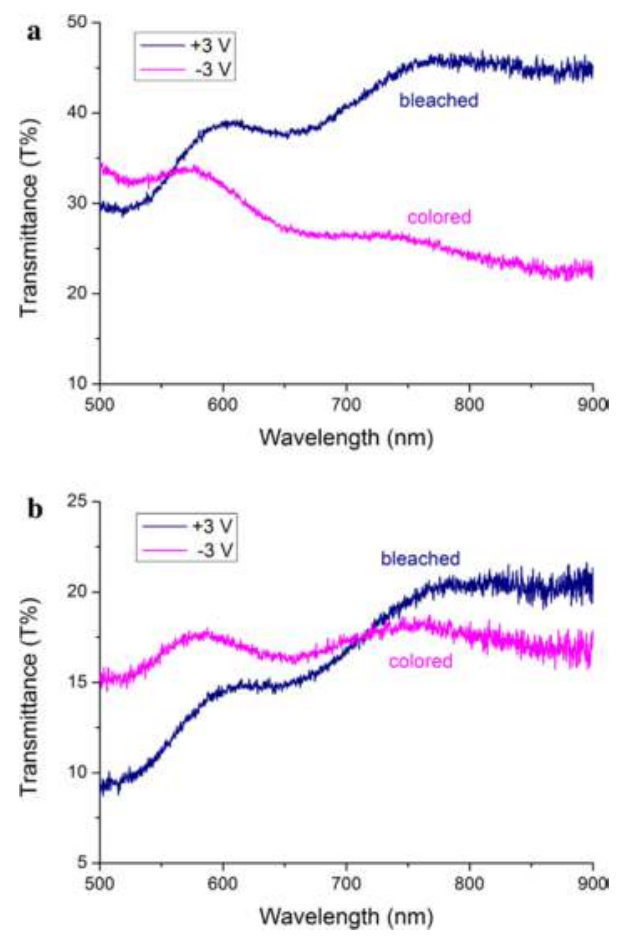

Figure 4. (a) Visible transmittance spectra of $\mathrm{WO}_{3}$ nanofiber based ECD with BMIMTFSI/PMMA/PC gel electrolyte under $+3 \mathrm{~V}$ and $-3 \mathrm{~V}$ applied potentials. (b) Visible transmittance spectra of $\mathrm{WO}_{3}$ nanofiber based ECD with ACN:PC:PMMA:LiClO 4 gel electrolyte under $+3 \mathrm{~V}$ and $-3 \mathrm{~V}$ applied potentials. 
Coloration efficiency ( $\mathrm{CE}$ or $\mathrm{h}$ ) is a pivotal parameter for judging the suitability of any redox switching compound for an electro- chromic application. It is defined as the ratio of optical density change (DOD) induced by injected charge per unit area (Q) and is given by $C E=D O D / Q$ $\mathrm{DOD}=\log (\mathrm{Tb} / \mathrm{Tc})$. Injected charge per unit area was obtained from chronoamperometry measurements by integrating the I- $t$ curve [21]. DOD value was found as 0.26 and calculated coloration efficiency was $64.58 \mathrm{~cm}^{2} / \mathrm{C}$ for the ECD with BMIMTFSI/PMMA/PC gel electrolyte. For comparison, transmit- tance change of $20 \%$ and coloration efficiency $(\mathrm{CE})$ of $36 \mathrm{~cm} 2 / \mathrm{C}$ in the visible range were obtained for sol-gel deposited $\mathrm{WO}_{3}$ thin film on flexible ITO:PET substrate [12]. The electrochromic coloration efficiency (CE) was found to be $34 \mathrm{~cm}^{2} / \mathrm{C}$ and optical contrast was found as $12 \%$ for fibrous WO3 thin films prepared by pulsed spray pyrolysis technique [22]. In another study, a CE of $39.24 \mathrm{~cm}^{2} / \mathrm{C}$ and transmittance variation of $28 \%$ was obtained for nanobrick-like $\mathrm{WO}_{3}$ thin films [10]. It is apparent that our value for coloration efficiency is superior and transmittance variation is comparable to the reported values.

To investigate stability of ECD, the cyclic voltammetry was carried out on as-grown tungsten oxide nanofibers/ITO glass based ECD. An applied triangular potential varies between 3.0 and _ $3.0 \mathrm{~V}$ (coloration) and back to $3.0 \mathrm{~V}$ (bleaching) at a sweep rate of $100 \mathrm{mV} / \mathrm{s}$. The voltammogram after a few initial cycles of stabilization and optimization is shown as solid circle in Fig. 5. The cathodic coloration reaction peak appears around the extreme value of the applied potential of $-0.5 \mathrm{~V}$, and the anodic peak of bleaching reaction is saturated around $0.9 \mathrm{~V}$ with respect to standard $\mathrm{Ag} / \mathrm{AgCl}$ electrode. After over one hundred cycles, the voltammogram shown as open triangle in Fig. 5, seems not show any significant difference from the initial one [23]. Nanostructured forms appear to be the most promising due to high surface to volume ratios that could allow the faster insertion/extraction processes with ions together with an improved optical modulations. As a result, most literature reports on $\mathrm{WO}_{3}$ nanostructures indicate that nanostructured $\mathrm{WO}_{3}$ outperforms conventional $\mathrm{WO}_{3}$ for electrochromic applications. Shim and Shung reported electrochromic properties of ultra long crystalline $\mathrm{WO}_{3}$ nanowires that can be directly prepared on an ITO-coated glass substrate using electrospinning method. The electrochromic and electrical prop- erties of the electrospun $\mathrm{WO}_{3}$ nanowire electrodes were investigated and compared with those of conventional $\mathrm{WO}_{3}$ thin film electrodes. As a result, the one-dimensional WO3 nanowires showed faster charge transfer and optical responses than the thin film electrodes. The coloration efficiency of the electrospun $\mathrm{WO}_{3}$ nanowires was also found to be greater than the thin film. The coloration efficiency of the electrospun WO3 nanofibers in our study is also greater $\left(64.58 \mathrm{~cm}^{2} / \mathrm{C}\right)$ by $18.58 \%$ than the sol-gel spin-coated thin film electrode $\left(46 \mathrm{~cm}^{2} / \mathrm{C}\right)$ [24]. The construction of solid-state $\mathrm{WO}_{3}$ nanofiber based ECD with good performance serves as a proof of concept that ionic liquid based gel electrolyte can be successfully applied in ECDs. This approach represents another field for perspective utilization of ionic liquids. The advantages of the proposed technology are as follow: high coloration efficiency (up to $64.58 \mathrm{~cm}^{2} / \mathrm{C}$ ), high working stability (100 cycles without change in electrochemical activity), optical contrast up to $20.82 \%$, simplicity of ECD's construction, benefit of nanofiber and possibility of their application in commercial fields. 


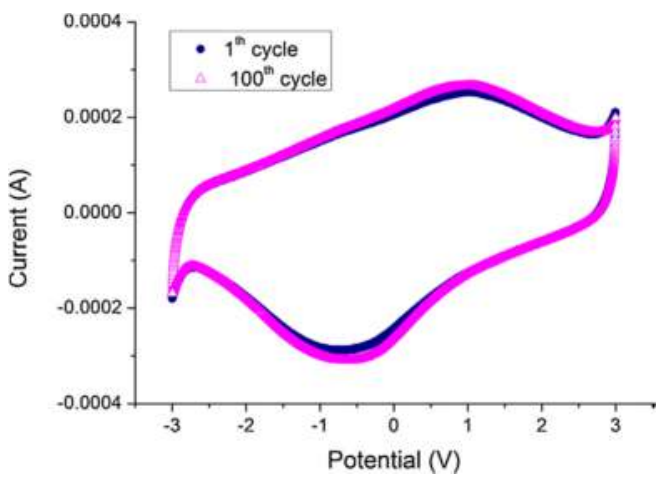

Figure 5. Cyclic voltammograms $\mathrm{WO}_{3}$ nanofiber based ECD after 1th and 100th cycles.

\section{Conclusions}

$\mathrm{WO}_{3}$ electrochromic nanofibers were successfully prepared on ITO coated glass at room temperature by electrospinning technique. $\mathrm{WO}_{3}$ nanofibers indicated good electrochromic properties switching reversibly from transparent to blue color when cycled in different ionic liquids and Li-PC based electrolyte. Electrochromic activity was confirmed building $\mathrm{WO}_{3}$ nanofiber solid state device using a ionic liquid/PMMA/PC plasticized gel electrolyte. This study essentially opens a whole avenue of ECD applications related with $\mathrm{WO}_{3}$ fibers.

\section{Acknowledgements}

The authors wish to acknowledge the TUBITAK Project-with number $114 Z 321$ for the financial support throughout the research.

\section{References}

[1] K. Tajima, H. Hotta, Y. Yamada, Sol. Energy Mater. Sol. Cells 104 (2012) 146-151.

[2] J. Zhang, X.L. Wang, Sol. Energy Mater. Sol. Cells 95 (2011) 2107-2112.

[3] D.Y. Ma, G.Y. Shi, H.Z. Wang, J. Mater. Chem. A 1 (2013) 684-691.

[4] G.F. Cai, D. Zhou, Q.Q. Xiong, Sol. Energy Mater. Sol. Cells 117 (2013) 231-238.

[5] Z.M. Huang, Y.Z. Zhang, M. Kotaki, Compos. Sci. Technol. 63 (2003) 2223-2253.

[6] B. Liu, E.S. Aydil, J. Am. Chem. Soc. 131 (2009) 3985-3990.

[7] S.V. Green, E. Pehlivan, C.G. Granqvist, Sol. Energy Mater. Sol. Cells 99 (2012) 339-344.

[8] M.M. El-Nahass, M.M. Saadeldin, Mater. Sci. Semicond. Process. 29 (2015) 201-205.

[9] S. Sallard, T. Brezesinski, J. Phys. Chem. C 111 (2007) 7200-7206.

[10] V.V. Kondalkar, R.R. Kharade, Superlattices Microstruct. 73 (2014) 290-295.

[11] P. Zeng, J. Zhai, Electrochem. Commun. 26 (2013) 5-9.

[12] A. Bessiere, J.C. Badot, Electrochim. Acta 46 (2001) 2251-2256.

[13] J. Wang, E. Khoo, J. Phys. Chem. C 113 (2009) 9655-9658.

[14] A.L. Dyer, C.R.G. Grenier, J.R. Reynolds, Adv. Funct. Mater. 17 (2007) 1480-1486.

[15] T.A. Nguyen, S. Park, Sens. Actuators B 160 (2011) 549-554.

[16] Y. Pang, X. Li, G. Shi, Thin Solid Films 516 (2008) 6512-6516.

[17] M. Kırıştı, F. Bozduman, A. Uygun Oksuz, L. Oksuz, A. Hala, Ind. Eng. Chem. Res.

53 (41) (2014) 15917-15922. 
[18] Z. Feng, D. Moa, Z. Wang, Electrochim. Acta 160 (2015) 160-168.

[19] G.F. Cai, J.P. Tu, D. Zhou, Sol. Energy Mater. Sol. Cells 124 (2014) 103-110.

[20] C. Dulgerbaki, A. Uygun Oksuz, Electroanal. 26 (2014) 2501-2512.

[21] H. Wei, X. Yan, J. Phys. Chem. C 116 (2012) 25052-25064.

[22] S.R. Bathe, P.S. Patil, Sol. Energy Mater. Sol. Cells 91 (2007) 1097-1101.

[23] C.C. Liao, F.R. Chen, Sol. Energy Mater. Sol. Cells 90 (2006) 1147-1155.

[24] H.S. Shim, J.W. Kim, Y.E. Sung, Sol. Energy Mater. Sol. Cells 93 (2009)

2062-2068. 